\title{
Three dimensional characterization of a silica hollow sphere with an iron oxide core by annular dark field scanning confocal electron microscopy
}

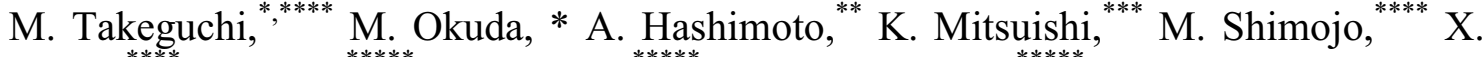

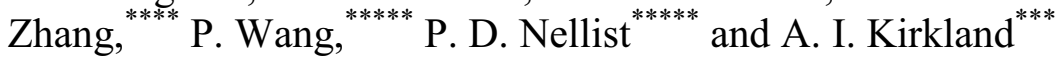 \\ * Nano-characterization Center, National Institute for Materials Science, 3-13 Sakura, \\ Tsukuba 305-003, Japan \\ ** International Center for Young Scientists, National Institute for Materials Science, \\ 1-2-1 Sengen, Tsukuba 305-0047, Japan \\ *** Quantum Dot Research Center, National Institute for Materials Science, 3-13 Sakura, \\ Tsukuba 305-003, Japan \\ ${ }^{* * * *}$ Advanced Science Research Laboratory, Saitama Institute of Technology, 1690 \\ Fusaiji, Fukuya, Saitama 369-0293, Japan \\ ${ }^{* * * * *}$ Department of Materials, University of Oxford, Parks Road, OX1 3PH Oxford, UK
}

Scanning confocal electron microscopy (SCEM) was first implement by N.J.Zaluzec et al. [1] and has recently become established as a three dimensional (3D) characterization tool by operating in energy filtered (EF) [2] and annular dark field (ADF) modes [3]. Since the 3D information transfer in these modes is improved compared with that of ADF-STEM and bright field (BF)-SCEM, the depth of field of not only point like objects but also laterally extended ones can be dramatically reduced [4-6]. Incoherent EF-SCEM has almost ideal 3D transfer function but inherently its signal is very small. On the other hand, although ADF-SCEM cannot be incoherent imaging and therefore its 3D transfer function is not ideal when low angle ADF electrons are collected, the depth of field of ADF-SCEM is capable of producing interpretable 3D images with sufficient signal intensity for various types of objects [6].

In the present work, we characterized 3D structure of silica hollow spheres with iron oxide cores by ADF-SCEM using an Oxford-JEOL 2200MCO equipped with aberration correctors for probe-forming and imaging lenses, pinhole, 3D stage scanning system and ADF aperture [3,7]. The sample was a rattle-type silica hollow sphere including an iron oxide $\left(\mathrm{Fe}_{2} \mathrm{O}_{3}\right)$ core prepared by the template method described by $\mathrm{Zhu}$ et al. [8].

Elemental maps taken by energy filtering are shown in Fig.1. It is obvious that iron covers the silica surface, but internal iron distribution cannot be clearly observed. Figure 2 shows (a) HAADF-STEM image and $(b, c)$ XY and XZ sliced images extracted from the $3 \mathrm{D}$ reconstruction through a computer tomography (CT) method. These were produced from a tilt-series of HAADF-STEM images from $-60^{\circ}$ to $+60^{\circ}$ at $2^{\circ}$ decrements. The acquisition time of the tilt-series was about 2 hours. The internal structure is clearly seen in the XY sliced image, but it is noted that the XZ sliced image exhibits missing-angle induced artifacts.

Figure 3 shows (a) HAADF-STEM image and (b,c) XY and XZ sliced images taken by ADF-SCEM. It was found that the core was also hollow, that is consistent with the CT observation results. Thus, it is demonstrated that ADF-SCEM enables us to observe sliced images of objects at desired positions directly. 


\section{References}

[1] N. J. Zaluzec, Microscopy- Today 6 (2003) 8.

[2] P. Wang et al., Microsc. Microanl. 15(Suppl.2) (2009) 42.

[3] A. Hashimoto et al., J. Appl. Phys. 106 (2009) 086101.

[4] H. L. Xin and D. A. Muller, J. Electron Microsc. 58 (2009) 157.

[5] A. J. D'Alfonso, et al., Ultramicroscopy 108 (2008) 1567.

[6] K. Mitsuishi et al., submitted.

[7] M. Takeguchi et al., J. Electron Microsc. 58 (2008) 123.

[8] Y. Zhu et al., Chem. Mater. 21 (2009) 2547.

[9] A part of this research was financially supported by Grant-in-Aid for Scientific Research and Bilateral Joint Research Project (Japan-UK) of JSPS.

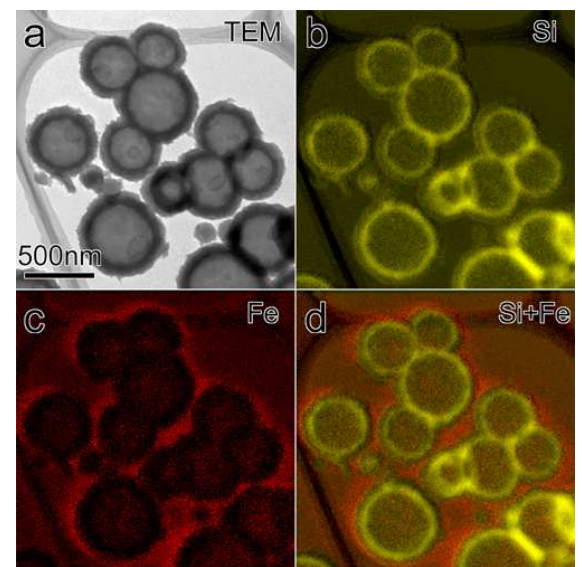

FIG.1 (a) HAADF-STEM image, (b) Si map, (C) Fe map, and (d) Si and Fe overlaid map of silica hollow spheres with iron oxide cores.

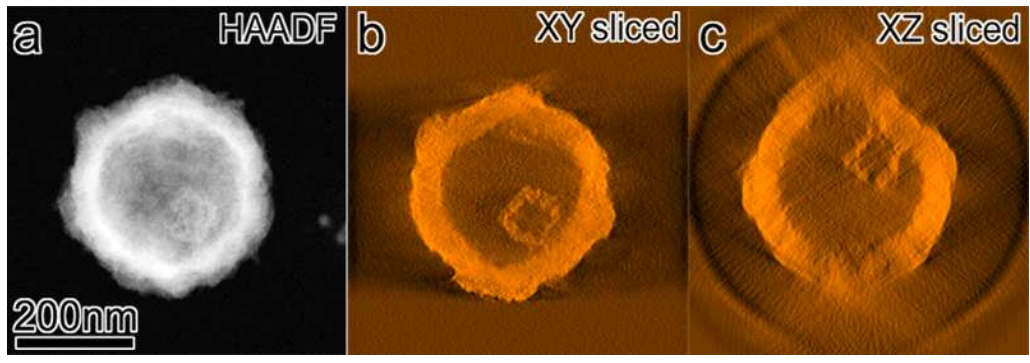

FIG.2 (a) HAADF-STEM image, and (b,c) XY and XZ sliced images extracted from TEM-CT 3D reconstruction of silica hollow spheres with iron oxide cores.

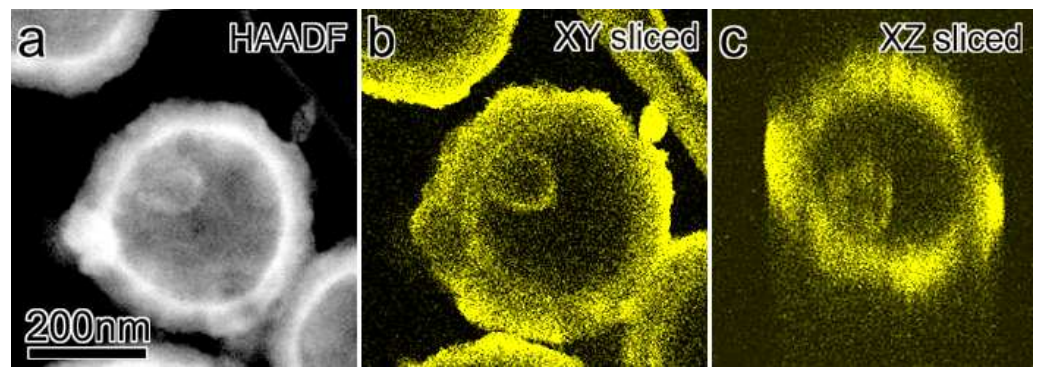

FIG.3 (a) HAADF-STEM image, (b,c) XY and XZ ADF-SCEM sliced images of silica hollow spheres with iron oxide cores. 